\title{
Cohomology of the Grothendieck construction
}

\author{
Teimuraz Pirashvili* and María Julia Redondo ${ }^{\dagger \ddagger}$
}

\begin{abstract}
We consider cohomology of small categories with coefficients in a natural system in the sense of Baues and Wirsching. For any functor $\mathrm{L}: \mathbf{K} \rightarrow \mathbf{C A T}$, we construct a spectral sequence abutting to the cohomology of the Grothendieck construction of $\mathrm{L}$ in terms of the cohomology of $\mathbf{K}$ and of $\mathrm{L}(k)$, for $k \in \mathrm{Ob} \mathbf{K}$.
\end{abstract}

2000 Mathematics Subject Classification : 18G40

\section{Introduction}

Given a functor $\mathrm{L}: \mathbf{K} \rightarrow \mathbf{C A T}$, there is a well defined category $\int_{\mathbf{K}} \mathrm{L}$ called the Grothendieck construction of L. Our aim is to construct a spectral sequence abutting to the cohomology of $\int_{\mathbf{K}} \mathrm{L}$ whose $\mathrm{E}_{2}$ term is given in terms of the cohomology of $\mathbf{K}$ and of $\mathrm{L}(k)$, for $k \in \mathrm{Ob} \mathbf{K}$.

If one takes the cohomology of small categories with constant coefficients then the existence of such spectral sequence is a consequence of Thomason's work [T].

In this paper we consider cohomology of small categories with coefficients in a natural system in the sense of Baues and Wirsching [BW]. For a natural system $\mathrm{D}$ on $\int_{\mathbf{K}} \mathrm{L}$ and an object $k$ of $\mathbf{K}$, there is an induced natural system $\mathrm{D}_{k}$ on $\mathrm{L}(k)$. We show that, under some condition on D (see Theorem 5.1), there is a convergent spectral sequence

$$
\mathrm{E}_{2}^{p q}=\mathrm{H}^{p}\left(\mathbf{K}, \mathbb{H}^{q}\left(\mathrm{~L}(.), \mathrm{D}_{(.)}\right)\right) \Rightarrow \mathrm{H}^{p+q}\left(\int_{\mathbf{K}} \mathrm{L}, \mathrm{D}\right),
$$

where $\mathbb{H}^{q}\left(\mathrm{~L}(),. \mathrm{D}_{(.)}\right)$is a contravariant functor on $\mathbf{K}$ which assigns to an object $k$ the group $\mathrm{H}^{q}\left(\mathrm{~L}(k), \mathrm{D}_{k}\right)$. We deduce this result from another spectral sequence (see

\footnotetext{
${ }^{*}$ T. Pirashvili; Razmadze Mathematical Institute of Georgian Academy of Sciences, Alexidze str. 1 Tbilisi, 0193, Republic of Georgia. E-mail address: pira@rmi.acnet.ge

${ }^{\dagger}$ M. J. Redondo; Instituto de Matemática, Universidad Nacional del Sur, Av. Alem 1253, (8000) Bahía Blanca, Argentina. E-mail address: mredondo@criba.edu.ar

${ }^{\ddagger}$ The first author was supported by DFG at University of Bielefeld; the second author is a researcher from CONICET, Argentina.
} 
Theorem 4.1) which holds for any natural system D but involves cohomology of some related categories $\tilde{\mathrm{L}}(k)$ instead of $\mathrm{L}(k)$.

Some particular cases have been already considered in the literature. For instance, if $\mathbf{K}$ is the one object category associated to a group $G$, and the natural system D comes from a bifunctor, the mentioned spectral sequence has been constructed in [CR], see Remark [5.3 Another particular case appears in [P], where $\mathbf{K}$ is arbitrary but $\mathrm{L}$ has values in sets, that is, in discrete categories.

\section{Baues-Wirsching cohomology}

Here we fix notation and we prove some elementary facts which will be needed later. For a small category $\mathbf{C}$ we let $\mathcal{F} \mathbf{C}$ denote the category of factorizations in $\mathbf{C}$ BW]. Objects of $\mathcal{F} \mathbf{C}$ are the morphisms in $\mathbf{C}$, and a morphism from $\alpha: x \rightarrow y$ to $\beta: u \rightarrow v$ is a pair $(\nu: u \rightarrow x, \psi: y \rightarrow v)$ of morphisms in $\mathbf{C}$ such that $\beta=\psi \alpha \nu$, that is, one has a commutative diagram

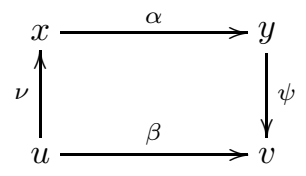

The composition in $\mathcal{F} \mathbf{C}$ is defined by $(\nu, \psi)\left(\nu^{\prime}, \psi^{\prime}\right)=\left(\nu^{\prime} \nu, \psi \psi^{\prime}\right)$. A natural system on $\mathbf{C}$ is just a covariant functor $\mathrm{D}: \mathcal{F} \mathbf{C} \rightarrow \mathbf{A b}$. Now, following [BW], one defines the cohomology $\mathrm{H}^{*}(\mathbf{C}, \mathrm{D})$ as the cohomology of the cochain complex $\mathrm{F}^{*}(\mathbf{C}, \mathrm{D})$ given by

$$
\mathrm{F}^{n}(\mathbf{C}, \mathrm{D})=\prod_{\substack{\alpha_{1} \ldots \ldots \alpha_{n} \\ c_{0} \stackrel{\alpha_{n}}{\leftarrow} c_{n}}} \mathrm{D}\left(\alpha_{1} \cdots \alpha_{n}\right)
$$

and the coboundary map

$$
d: \mathrm{F}^{n}(\mathbf{C}, \mathrm{D}) \rightarrow \mathrm{F}^{n+1}(\mathbf{C}, \mathrm{D})
$$

is given by

$$
\begin{aligned}
(d f)\left(\alpha_{1}, \cdots, \alpha_{n+1}\right) & =\left(\alpha_{1}\right)_{*} f\left(\alpha_{2}, \cdots, \alpha_{n+1}\right) \\
& +\sum_{i=1}^{n}(-1)^{i} f\left(\alpha_{1}, \cdots, \alpha_{i} \alpha_{i+1}, \cdots, \alpha_{n+1}\right) \\
& +(-1)^{n+1}\left(\alpha_{n+1}\right)^{*} f\left(\alpha_{1}, \cdots, \alpha_{n}\right) .
\end{aligned}
$$

Here, and in the rest of the paper, we use the following convention. For $\alpha: x \rightarrow y$ and elements $a \in \mathrm{D}(u, x), b \in \mathrm{D}(y, v)$, we write $\alpha_{*} a$ and $\alpha^{*} b$ for the image of the elements $a$ and $b$ by the homomorphisms $\mathrm{D}\left(i d_{u}, \alpha\right): \mathrm{D}(u, x) \rightarrow \mathrm{D}(u, y)$ and $\mathrm{D}\left(\alpha, i d_{v}\right): \mathrm{D}(y, v) \rightarrow \mathrm{D}(x, v)$ respectively. 
Consider the natural functors

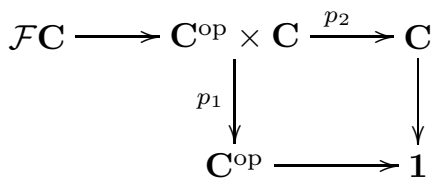

where the first top functor sends an arrow $\alpha: c \rightarrow d$ to the pair $(c, d), p_{1}$ and $p_{2}$ are projections and, finally, $\mathbf{1}$ is the category with one object and one arrow. Then one gets that any bifunctor, covariant, contravariant functor or any abelian group gives rise to a natural system, and then Baues-Wirsching cohomology generalizes known theories.

Natural systems coming from the functor $\mathbf{1} \rightarrow \mathbf{A b}$ are called constant. In this case, the cohomology of $\mathbf{C}$ is the same as the cohomology of the corresponding classifying space.

We will need the following easy and well-known result.

LEMMA 2.1 Assume $\mathbf{C}$ has an initial object and $\mathrm{D}$ is constant. Then

$$
\mathrm{H}^{n}(\mathbf{C}, \mathrm{D})= \begin{cases}0, & \text { if } n>0, \\ \mathrm{D}, & \text { if } n=0 .\end{cases}
$$

Proof. It follows from the fact that the classifying space BC is contractible, see Q, §1, Corollary 2].

The following result is a specialization of [FFPS, Lemma 1.5, page 10], setting $\mathbf{C}=\mathcal{F} \mathcal{A}, \mathbf{D}=\mathcal{F} \mathcal{B}$ and $\mathrm{F}$ the constant natural system on $\mathbf{C}$ with value $\mathbb{Z}$.

LEMMA 2.2 Let $(l, r)$ be an adjoint pair from $\mathcal{A}$ to $\mathcal{B}$. Then for any natural system $G$ on $\mathcal{B}$ one has the natural isomorphism

$$
\mathrm{H}^{*}\left(\mathcal{A}, l^{*}(G)\right) \simeq \mathrm{H}^{*}(\mathcal{B}, G) .
$$

Proof. Observe first that the induced pair $(\mathcal{F}(l), \mathcal{F}(r))$ from $\mathcal{F} \mathcal{A}$ to $\mathcal{F B}$ is also an adjoint pair. Then we can use formal adjunction.

However we need more sophisticated relationship between cohomologies of categories involved in adjoint situation. The following result is a bit more general than one proved recently by Muro (see [M] Theorem 6.5]); his proof uses explicit homotopies, which he constructs using 2-categories. Here we use completely different ideas.

PROPOSITION 2.3 Let $(l, r)$ be an adjoint pair from $\mathbf{C}$ to $\mathbf{C}^{\prime}$. Assume $\mathrm{E}$ is a natural system on $\mathbf{C}$, and let $\tilde{\mathrm{E}}$ and $\overline{\mathrm{E}}$ be the natural systems, on $\mathbf{C}$ and $\mathbf{C}^{\prime}$ respectively, defined by

$$
\begin{aligned}
& \tilde{\mathrm{E}}(c \stackrel{\alpha}{\rightarrow} d):=\mathrm{E}\left(\epsilon_{d} \circ \alpha\right), \\
& \overline{\mathrm{E}}(u \stackrel{\beta}{\rightarrow} v):=\mathrm{E}(r(u) \stackrel{r(\beta)}{\longrightarrow} r(v)),
\end{aligned}
$$


where $\epsilon: i d_{\mathbf{C}} \rightarrow r l$ is the unit of the adjunction. Then

$$
\mathrm{H}^{*}\left(\mathbf{C}^{\prime}, \overline{\mathrm{E}}\right) \simeq \mathrm{H}^{*}(\mathbf{C}, \tilde{\mathrm{E}}) .
$$

Proof. Varying E in the category of all natural systems defined on $\mathbf{C}$, one obtains two sequences of functors

$$
\left(\mathrm{E} \mapsto \mathrm{H}^{n}(\mathbf{C}, \tilde{\mathrm{E}})\right)_{n \geq 0}
$$

and

$$
\left(\mathrm{E} \mapsto \mathrm{H}^{n}(\mathbf{C}, \overline{\mathrm{E}})\right)_{n \geq 0}
$$

such that for any short exact sequence of natural systems on $\mathbf{C}$

$$
0 \rightarrow \mathrm{E}_{1} \rightarrow \mathrm{E} \rightarrow \mathrm{E}_{2} \rightarrow 0
$$

there are corresponding long exact cohomological sequences in both theories. Moreover cohomology respects products, therefore it suffices to show that both theories vanish on injective cogenerators in positive dimensions and have same values in dimension zero.

Let us recall that in a category of functors, the injective cogenerators are "duals" of representable functors. Therefore in the category of natural systems on $\mathbf{C}$, the injective cogenerators are natural systems $\mathrm{J}_{\mu}$, where $\mu: a \rightarrow b$ runs over all morphisms in $\mathbf{C}$ and, for any morphism $\alpha: c \rightarrow d$, one has

$$
\mathrm{J}_{\mu}(\alpha)=\operatorname{Maps}\left(S_{\mu}(\alpha), \mathbb{Q} / \mathbb{Z}\right)
$$

where $S_{\mu}: \mathcal{F} \mathbf{C}^{\text {op }} \rightarrow$ Sets is given by

$$
S_{\mu}(\alpha)=\operatorname{Hom}_{\mathcal{F} \mathbf{C}}(\alpha, \mu)=\{a \stackrel{\nu}{\longrightarrow} c, d \stackrel{\psi}{\longrightarrow} b \mid \psi \alpha \nu=\mu\} .
$$

One has an obvious isomorphism of cochain complexes

$$
\mathrm{F}^{*}\left(\mathbf{C}, \tilde{\mathrm{J}}_{\mu}\right) \simeq \mathrm{F}^{*}\left(\mathbf{C}_{\mu}, \mathbb{Q} / \mathbb{Z}\right)
$$

where on the right hand side the cohomology is taken with constant coefficients, while $\mathbf{C}_{\mu}$ is the category whose objects are pairs

$$
(a \stackrel{\nu}{\longrightarrow} c, r l(c) \stackrel{\psi}{\longrightarrow} b)
$$

such that $\psi \circ \epsilon_{c} \circ \nu=\mu$, while a morphism from $(\nu, \psi)$ to $\left(\nu^{\prime}, \psi^{\prime}\right)$ is a morphism $\beta: c \rightarrow c^{\prime}$ such that the following diagrams
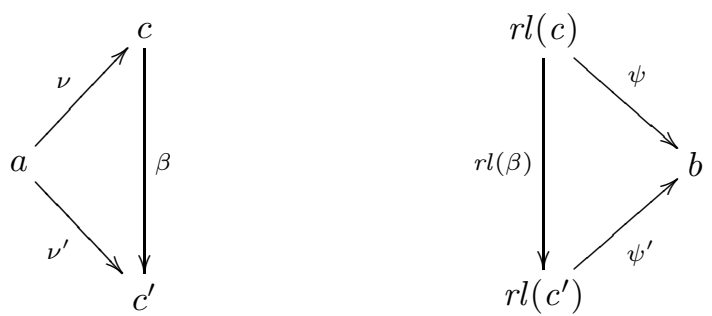
commute. Let us observe also that $r l(\beta) \circ \epsilon_{c}=\epsilon_{c^{\prime}} \circ \beta$, since $\epsilon$ is a natural transformation. Now let $\Delta_{\mu}$ be the set of morphisms $\gamma: \operatorname{rl}(a) \rightarrow b$ such that $\gamma \circ \epsilon_{a}=\mu$. For any $\gamma \in \Delta_{\mu}$ we let $\mathbf{C}_{\mu, \gamma}$ be the full subcategory of $\mathbf{C}_{\mu}$ consisting of objects $(\nu, \psi)$ such that $\psi \circ \operatorname{rl}(\nu)=\gamma$. It is clear that $\mathbf{C}_{\mu, \gamma} \cap \mathbf{C}_{\mu, \gamma^{\prime}}=\emptyset$ provided $\gamma \neq \gamma^{\prime}$. It is also clear that for any object $(\nu, \psi) \in \mathbf{C}_{\mu}$ one has that $(\nu, \psi) \in \mathbf{C}_{\mu, \gamma}$ for $\gamma=\psi \circ \operatorname{rl}(\nu)$. Thus $\mathbf{C}_{\mu}$ is the disjoint union

$$
\mathbf{C}_{\mu}=\bigsqcup_{\gamma \in \Delta_{\mu}} \mathbf{C}_{\mu, \gamma}
$$

Since for each $\gamma \in \Delta_{\mu},\left(i d_{a}, \gamma\right)$ is an initial object in $\mathbf{C}_{\mu, \gamma}$, we obtain

$$
\mathrm{H}^{n}\left(\mathbf{C}, \tilde{\mathrm{J}}_{\mu}\right)= \begin{cases}0, & n>0 \\ \mathbb{Z}\left[\Delta_{\mu}\right], & n=0 .\end{cases}
$$

Next we consider the cohomology $\mathrm{H}^{*}\left(\mathbf{C}^{\prime}, \overline{\mathrm{J}}_{\mu}\right)$. Since

$$
\mathrm{F}^{p}\left(\mathbf{C}^{\prime}, \overline{\mathrm{J}}_{\mu}\right)=\prod_{\substack{\omega_{1} \\ u_{0} \ldots \ldots \omega_{p}}} \overline{\mathrm{J}}_{\mu}\left(\omega_{1} \circ \cdots \circ \omega_{p}\right)
$$

and $\overline{\mathrm{J}}_{\mu}\left(\omega_{1} \circ \cdots \circ \omega_{p}\right)=\prod \mathbb{Q} / \mathbb{Z}$, where the product is taken over all morphisms $r\left(\omega_{1} \circ \cdots \circ \omega_{p}\right) \rightarrow \mu$ in $\mathcal{F} \mathbf{C}$, it follows that one has an isomorphism of complexes

$$
\mathrm{F}^{*}\left(\mathbf{C}^{\prime}, \overline{\mathrm{J}}_{\mu}\right) \simeq \mathrm{F}^{*}\left(\mathbf{C}_{\mu}^{\prime}, \mathbb{Q} / \mathbb{Z}\right)
$$

where on the right hand side the cohomology is taken with constant coefficients and $\mathbf{C}_{\mu}^{\prime}$ is the category whose objects are triples

$$
(u, a \stackrel{\nu}{\longrightarrow} r(u), r(u) \stackrel{\psi}{\longrightarrow} b)
$$

such that $\psi \circ \nu=\mu$, while a morphism from $(u, \nu, \psi)$ to $\left(u^{\prime}, \nu^{\prime}, \psi^{\prime}\right)$ is a morphism $\tau: u \rightarrow u^{\prime}$ such that the following diagram

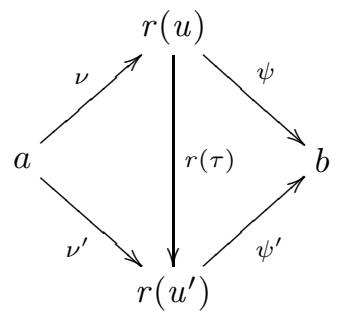

commutes. By adjointness, for any $\nu: a \rightarrow r(u)$ there exists a unique $\hat{\nu}: l(a) \rightarrow u$ such that the diagram

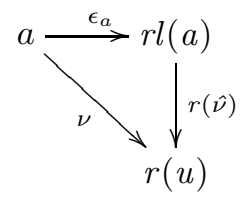


commutes. Then it follows that $\mathbf{C}_{\mu}^{\prime}$ has the following equivalent description: objects are triples

$$
(u, l(a) \stackrel{\hat{\nu}}{\longrightarrow} u, r(u) \stackrel{\psi}{\longrightarrow} b)
$$

such that $\mu=\psi \circ r(\hat{\nu}) \circ \epsilon_{a}$. Recall that $\Delta_{\mu}$ is the set of morphisms $\gamma: \operatorname{rl}(a) \rightarrow b$ such that $\gamma \circ \epsilon_{a}=\mu$. So, for any $\gamma \in \Delta_{\mu}$ we let $\mathbf{C}_{\mu, \gamma}^{\prime}$ be the full subcategory of $\mathbf{C}_{\mu}^{\prime}$ consisting of objects $(u, \hat{\nu}, \psi)$ such that $\psi \circ r(\hat{\nu})=\gamma$. It is clear that $\mathbf{C}_{\mu, \gamma}^{\prime} \cap \mathbf{C}_{\mu, \gamma^{\prime}}^{\prime}=$ $\emptyset$ provided $\gamma \neq \gamma^{\prime}$. It is also clear that for any object $(u, \hat{\nu}, \psi) \in \mathbf{C}_{\mu}^{\prime}$ one has that $(u, \hat{\nu}, \psi) \in \mathbf{C}_{\mu, \gamma}^{\prime}$ for $\gamma=\psi \circ r(\hat{\nu})$. Thus $\mathbf{C}_{\mu}^{\prime}$ is the disjoint union

$$
\mathbf{C}_{\mu}^{\prime}=\bigsqcup_{\gamma \in \Delta_{\mu}} \mathbf{C}_{\mu, \gamma}^{\prime}
$$

Since for each $\gamma \in \Delta_{\mu},\left(l(a), i d_{l(a)}, \gamma\right)=\left(l(a), \hat{\epsilon}_{a}, \gamma\right)$ is an initial object in $\mathbf{C}_{\mu, \gamma}^{\prime}$, we obtain

$$
\mathrm{H}^{n}\left(\mathbf{C}^{\prime}, \overline{\mathrm{J}}_{\mu}\right)= \begin{cases}0, & n>0 \\ \mathbb{Z}\left[\Delta_{\mu}\right], & n=0\end{cases}
$$

We encountered the following lemma, which we think is of independent interest, when looking for the proof of the previous proposition.

Let $a$ be an object of a small category $\mathbf{C}$ and let $\mathrm{T}: \mathbf{C}^{o p} \rightarrow$ Sets be a functor. For any $m \in \mathrm{T}(a)$ we define a functor

$$
S_{a, \mathrm{~T}, m}: \mathcal{F} \mathbf{C}^{o p} \rightarrow \text { Sets }
$$

using the following rule:

$$
S_{a, \mathrm{~T}, m}(\alpha: c \rightarrow d)=\left\{(a \stackrel{\eta}{\longrightarrow} c ; r \in \mathrm{T}(d)) \mid \eta^{*} \alpha^{*}(r)=m\right\} .
$$

For any abelian group $A$ we put

$$
\mathrm{D}_{a, \mathrm{~T}, m, A}(\alpha: c \rightarrow d)=\operatorname{Maps}\left(S_{a, \mathrm{~T}, m}(\alpha), A\right) .
$$

Then $\mathrm{D}_{a, \mathrm{~T}, m, A}: \mathcal{F} \mathbf{C} \rightarrow \mathbf{A b}$ is a natural system.

LEMMA 2.4 For any small category $\mathbf{C}$, any functor $\mathrm{T}: \mathbf{C}^{o p} \rightarrow$ Sets, any element $m \in \mathrm{T}(a)$ and any abelian group $A$, one has

$$
\mathrm{H}^{n}\left(\mathbf{C}, \mathrm{D}_{a, \mathrm{~T}, m, A}\right)= \begin{cases}0, & n>0 \\ A, & n=0 .\end{cases}
$$

Proof. Consider the category $\mathbf{C}_{a, \mathrm{~T}, m}$ whose objects are pairs $(\eta: a \rightarrow d, r \in$ $\mathrm{T}(d))$, with $\eta^{*}(r)=m$. A morphism from $(\eta, r)$ to $\left(\eta^{\prime}, r^{\prime}\right)$ in $\mathbf{C}_{a, \mathrm{~T}, m}$ is a morphism $\beta: d \rightarrow d^{\prime}$ such that $\beta \eta=\eta^{\prime}$ and $\beta^{*}\left(r^{\prime}\right)=r$. Then one has an isomorphism of complexes

$$
\mathrm{F}^{*}\left(\mathbf{C}, \mathrm{D}_{a, \mathrm{~T}, m, A}\right) \cong \mathrm{F}^{*}\left(\mathbf{C}_{a, \mathrm{~T}, m}, A\right) .
$$

Since $\left(i d_{a}, m\right)$ is an initial object of $\mathbf{C}_{a, \mathrm{~T}, m}$ the result follows. 


\section{Grothendieck construction and Thomason's func- tor}

Now we recall the classical construction due to Grothendieck [SGA1 Exposé VI.8]. Let CAT be the category of all small categories. For any small category $\mathbf{K}$, and any functor

$$
\mathrm{L}: \mathbf{K} \rightarrow \mathbf{C A T}
$$

the category $\int_{\mathbf{K}} \mathrm{L}$ is defined as follows: objects are pairs $(k, x)$ with $k \in \mathrm{Ob} \mathbf{K}$ and $x \in \mathrm{ObL}(k)$. A morphism $\left(k_{0}, x_{0}\right) \rightarrow\left(k_{1}, x_{1}\right)$ in $\int_{\mathbf{K}} \mathrm{L}$ is a pair $(\alpha, \xi)$ where $\alpha: k_{0} \rightarrow k_{1}$ is a morphism in $\mathbf{K}$ and $\xi: \mathrm{L}(\alpha)\left(x_{0}\right) \rightarrow x_{1}$ is a morphism in $\mathrm{L}\left(k_{1}\right)$. Composition law is given by

$$
(\alpha, \xi)\left(\alpha^{\prime}, \xi^{\prime}\right)=\left(\alpha \alpha^{\prime}, \xi \circ \mathrm{L}(\alpha)\left(\xi^{\prime}\right)\right)
$$

Let $\mathrm{L}: \mathbf{K} \rightarrow \mathbf{C A T}$ be a functor. In [T Definition 1.2.2], Thomason defines a new functor

$$
\tilde{\mathrm{L}}: \mathbf{K} \rightarrow \mathbf{C A T}
$$

as follows. For any object $k$ in $\mathbf{K}$, the category $\tilde{\mathrm{L}}(k)$ has as objects pairs $(\alpha, x)$ where $\alpha: l \rightarrow k$ is a morphism in $\mathbf{K}$ and $x$ is an object in $\mathrm{L}(l)$. A morphism $(\alpha, x) \rightarrow\left(\alpha^{\prime}, x^{\prime}\right)$ in $\tilde{\mathrm{L}}(k)$ is a pair $(\beta, \xi)$ with $\beta: l \rightarrow l^{\prime}$ and $\xi: \mathrm{L}(\beta)(x) \rightarrow x^{\prime}$, such that the diagram

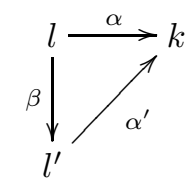

commutes. Composition is given by

$$
(\beta, \xi)\left(\beta^{\prime}, \xi^{\prime}\right)=\left(\beta \beta^{\prime}, \xi \circ \mathrm{L}(\beta)\left(\xi^{\prime}\right)\right)
$$

It is clear that $\tilde{\mathrm{L}}(k)$ is a well defined category. Moreover, if $\gamma: k \rightarrow k^{\prime}$ is a morphism in $\mathbf{K}$, then we have a functor

$$
\tilde{\mathrm{L}}(\gamma): \tilde{\mathrm{L}}(k) \rightarrow \tilde{\mathrm{L}}\left(k^{\prime}\right)
$$

given by

$$
\begin{aligned}
& \tilde{\mathrm{L}}(\gamma)(\alpha, x)=(\gamma \alpha, x), \\
& \tilde{\mathrm{L}}(\gamma)(\beta, \xi)=(\beta, \xi)
\end{aligned}
$$

for any $(\alpha, x) \in \operatorname{Ob} \tilde{\mathrm{L}}(k),(\beta, \xi) \in \operatorname{Mor} \tilde{\mathrm{L}}(k)$. In this way we get a functor $\tilde{\mathrm{L}}: \mathbf{K} \rightarrow$ CAT. 


\section{The first spectral sequence}

Keeping the notation of the preceding section, consider for each object $k$ in $\mathbf{K}$, the forgetful functor

$$
i_{k}: \tilde{\mathrm{L}}(k) \rightarrow \int_{\mathbf{K}} \mathrm{L}
$$

given on objects by

$$
i_{k}(\alpha: l \rightarrow k, x)=(l, x)
$$

and on morphisms by

$$
i_{k}(\beta, \xi)=(\beta, \xi) .
$$

If $\mathrm{D}$ is a natural system on $\int_{\mathbf{K}} \mathrm{L}$, the functor $i_{k}$ induces a natural system $i_{k}^{*} \mathrm{D}$ on $\tilde{\mathrm{L}}(k)$, for any $k \in \mathrm{Ob} \mathbf{K}$. Thus we can consider the cohomology $\mathrm{H}^{*}\left(\tilde{\mathrm{L}}(k), i_{k}^{*} \mathrm{D}\right)$. For any morphism $\gamma: k \rightarrow k^{\prime}$ in $\mathbf{K}$ one has a commutative diagram of functors

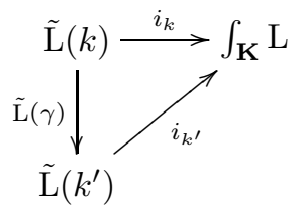

In particular, $i_{k}^{*} \mathrm{D}=\tilde{\mathrm{L}}(\gamma)^{*} i_{k^{\prime}}^{*} \mathrm{D}$, and therefore we have a well defined homomorphism in cohomology

$$
\mathrm{H}^{*}\left(\tilde{\mathrm{L}}(k), i_{k}^{*} \mathrm{D}\right) \rightarrow \mathrm{H}^{*}\left(\tilde{\mathrm{L}}\left(k^{\prime}\right), i_{k^{\prime}}^{*} \mathrm{D}\right) .
$$

In fact, we have a functor,

$$
\mathbb{H}^{*}\left(\tilde{\mathrm{L}}(.), i_{(.)}^{*} \mathrm{D}\right): \mathbf{K}^{\mathrm{op}} \rightarrow \mathbf{A b},
$$

sending $k \in \mathrm{Ob}(\mathbf{K})$ to $\mathrm{H}^{*}\left(\tilde{\mathrm{L}}(k), i_{k}^{*} \mathrm{D}\right)$.

THEOREM 4.1 Let $\mathbf{K}$ be a small category, $\mathrm{L}: \mathbf{K} \rightarrow \mathbf{C A T}$ a functor and $\mathrm{D} a$ natural system on $\int_{\mathbf{K}} \mathrm{L}$. Then there is a first quadrant spectral sequence

$$
\mathrm{E}_{2}^{p q}=\mathrm{H}^{p}\left(\mathbf{K}, \mathbb{H}^{q}\left(\tilde{\mathrm{L}}(.), i_{(.)}^{*} \mathrm{D}\right) \Rightarrow \mathrm{H}^{p+q}\left(\int_{\mathbf{K}} \mathrm{L}, \mathrm{D}\right) .\right.
$$

Proof. The proof is inspired by an argument given by Thomason in T, Lemma 1.2.4]. Let $C^{* *}$ be the bicomplex defined as follows. For any $p, q \geq 0$ let $M^{p, q}$ be the set of all triples

$$
\left(i_{0} \stackrel{\alpha_{1}}{\leftarrow} \cdots \stackrel{\alpha_{p}}{\leftarrow} i_{p} ;\left(j_{0}, x_{0}\right) \stackrel{\left(\beta_{1}, \xi_{1}\right)}{\longleftarrow} \ldots \stackrel{\left(\beta_{q}, \xi_{q}\right)}{\longleftarrow}\left(j_{q}, x_{q}\right) ; i_{p} \stackrel{\gamma}{\leftarrow} j_{0}\right)
$$

consisting of $p$ composable morphisms $\alpha_{1}, \cdots, \alpha_{p}$ in $\mathbf{K}, q$ composable morphisms $\left(\beta_{1}, \xi_{1}\right), \cdots,\left(\beta_{q}, \xi_{q}\right)$ in $\int_{\mathbf{K}} \mathrm{L}$, and a morphism in $\mathbf{K}$ connecting the first component of the target of $\left(\beta_{1}, \xi_{1}\right)$ with the source of $\alpha_{p}$. Now we set

$$
C^{p, q}=\prod \mathrm{D}\left(\left(\beta_{1}, \xi_{1}\right) \circ \cdots \circ\left(\beta_{q}, \xi_{q}\right)\right)
$$


where the product is taken over the set $M^{p, q}$. The homomorphisms

$$
C^{p, q} \stackrel{\delta^{p, q}}{\longrightarrow} C^{p+1, q} \quad \text { and } \quad C^{p, q} \stackrel{\partial^{p, q}}{\longrightarrow} C^{p, q+1}
$$

are given by

$$
\begin{aligned}
& \delta^{p, q}(f)\left(\alpha_{1}, \cdots, \alpha_{p+1} ;\left(\beta_{1}, \xi_{1}\right), \cdots,\left(\beta_{q}, \xi_{q}\right) ; \gamma\right)= \\
& =f\left(\alpha_{2}, \cdots, \alpha_{p+1} ;\left(\beta_{1}, \xi_{1}\right), \cdots,\left(\beta_{q}, \xi_{q}\right) ; \gamma\right) \\
& +\sum_{t=1}^{p}(-1)^{t} f\left(\alpha_{1}, \cdots, \alpha_{t} \alpha_{t+1}, \cdots, \alpha_{p+1} ;\left(\beta_{1}, \xi_{1}\right), \cdots,\left(\beta_{q}, \xi_{q}\right) ; \gamma\right) \\
& +(-1)^{p+1} f\left(\alpha_{1}, \cdots, \alpha_{p} ;\left(\beta_{1}, \xi_{1}\right), \cdots,\left(\beta_{q}, \xi_{q}\right) ; \alpha_{p+1} \gamma\right)
\end{aligned}
$$

and

$$
\begin{aligned}
& \partial^{p, q}(f)\left(\alpha_{1}, \cdots, \alpha_{p} ;\left(\beta_{1}, \xi_{1}\right), \cdots,\left(\beta_{q+1}, \xi_{q+1}\right) ; \gamma\right)= \\
& =\left(\beta_{1}, \xi_{1}\right)_{*} f\left(\alpha_{1}, \cdots, \alpha_{p} ;\left(\beta_{2}, \xi_{2}\right), \cdots,\left(\beta_{q+1}, \xi_{q+1}\right) ; \gamma \beta_{1}\right) \\
& +\sum_{t=1}^{q}(-1)^{t} f\left(\alpha_{1}, \cdots, \alpha_{p} ;\left(\beta_{1}, \xi_{1}\right), \cdots,\left(\beta_{t}, \xi_{t}\right)\left(\beta_{t+1}, \xi_{t+1}\right), \cdots,\left(\beta_{q+1}, \xi_{q+1}\right) ; \gamma\right) \\
& +(-1)^{q+1}\left(\beta_{q+1}, \xi_{q+1}\right)^{*} f\left(\alpha_{1}, \cdots, \alpha_{p} ;\left(\beta_{1}, \xi_{1}\right), \cdots,\left(\beta_{q}, \xi_{q}\right) ; \gamma\right) .
\end{aligned}
$$

Let $\operatorname{Tot}\left(C^{* *}\right)$ be the total complex associated to the bicomplex $C^{* *}=\left(C^{p, q}, \delta^{p, q},(-1)^{p} \partial^{p, q}\right)$. First we define the morphism of complexes

$$
\phi^{*}: \mathrm{F}^{*}\left(\int_{\mathbf{K}} \mathrm{L}, \mathrm{D}\right) \rightarrow C^{0, *}
$$

using the following formula

$$
\phi^{q}(f)\left(i_{0} ;\left(j_{0}, x_{0}\right) \stackrel{\left(\beta_{1}, \xi_{1}\right)}{\longleftarrow} \cdots \stackrel{\left(\beta_{q}, \xi_{q}\right)}{\longleftarrow}\left(j_{q}, x_{q}\right) ; i_{0} \stackrel{\gamma}{\leftarrow} j_{0}\right)=f\left(\left(\beta_{1}, \xi_{1}\right) \circ \cdots \circ\left(\beta_{q}, \xi_{q}\right)\right) .
$$

Since the composition

$$
\mathrm{F}^{*}\left(\int_{\mathbf{K}} \mathrm{L}, \mathrm{D}\right) \stackrel{\phi^{*}}{\longrightarrow} C^{0, *} \stackrel{\delta^{0, *}}{\longrightarrow} C^{1, *}
$$

vanishes, $\phi^{*}$ induces a homomorphism of complexes $\overline{\phi^{*}}: \mathrm{F}^{*}\left(\int_{\mathbf{K}} \mathrm{L}, \mathrm{D}\right) \rightarrow \operatorname{Tot}^{*}\left(C^{* *}\right)$. We claim that $\overline{\phi^{*}}$ is a quasi-isomorphism. Indeed, it suffices to show that for each $n \geq 0$ the sequence

$$
0 \rightarrow \mathrm{F}^{n}\left(\int_{\mathbf{K}} \mathrm{L}, \mathrm{D}\right) \rightarrow C^{0, n} \stackrel{\delta^{0, n}}{\longrightarrow} C^{1, n} \stackrel{\delta^{1, n}}{\longrightarrow} C^{2, n} \stackrel{\delta^{2, n}}{\longrightarrow} \cdots
$$

is exact. Note that the complex $C^{*, n}$ can be represented as the product over all $n$-strings

$$
\left(j_{0}, x_{0}\right) \stackrel{\left(\beta_{1}, \xi_{1}\right)}{\longleftarrow} \cdots \stackrel{\left(\beta_{n}, \xi_{n}\right)}{\longleftarrow}\left(j_{n}, x_{n}\right)
$$


of the complexes

$$
\mathrm{F}^{*}\left(\mathbf{K} \uparrow j_{0}, D\left(\left(\beta_{1}, \xi_{1}\right) \circ \cdots \circ\left(\beta_{n}, \xi_{n}\right)\right)\right),
$$

where $\mathbf{K} \uparrow j_{0}$ is the category whose objects are morphisms $j \stackrel{\sigma}{\leftarrow} j_{0}$ in $\mathbf{K}$ and morphisms $\sigma \rightarrow \sigma^{\prime}$ are morphisms $\tau: j \rightarrow j^{\prime}$ in $\mathbf{K}$ such that the following diagram commutes

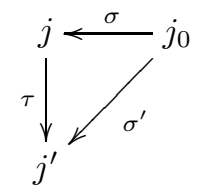

Let us observe that the coefficient $D\left(\left(\beta_{1}, \xi_{1}\right) \cdots\left(\beta_{n}, \xi_{n}\right)\right)$ is constant and $\mathbf{K} \uparrow j_{0}$ has an initial object $i d_{j_{0}}$, so the claim follows from Lemma 2.1. Now, it follows from the spectral sequence of a bicomplex that to finish the proof we only need to prove that

$$
\mathrm{H}^{*}\left(C^{p, *}\right)=\mathrm{F}^{p}\left(\mathbf{K}, \mathbb{H}^{*}\left(\tilde{\mathrm{L}}(.), i_{(.)}^{*} \mathrm{D}\right) .\right.
$$

But $C^{p, *}$ is the product over all $p$-strings $i_{0} \stackrel{\alpha_{1}}{\leftarrow} \ldots \stackrel{\alpha_{p}}{\leftarrow} i_{p}$ of the complexes $\mathrm{F}^{*}\left(\tilde{\mathrm{L}}\left(i_{p}\right), i_{i_{p}}^{*} \mathrm{D}\right)$, and the result follows.

\section{The second spectral sequence}

Let $\mathbf{K}$ be a small category and let $\mathrm{L}: \mathbf{K} \rightarrow \mathbf{C A T}$ be a functor as above. For each object $k \in \mathrm{Ob} \mathbf{K}$, one has an adjoint pair $\left(l_{k}, r_{k}\right)$ from $\tilde{\mathrm{L}}(k)$ to $\mathrm{L}(k)$ given by

$$
l_{k}(\alpha, x)=\mathrm{L}(\alpha)(x) \quad \text { and } \quad r_{k}(y)=\left(i d_{k}, y\right)
$$

for any $(l \stackrel{\alpha}{\rightarrow} k, x \in \mathrm{ObL}(l)) \in \mathrm{Ob} \tilde{\mathrm{L}}(k)$ and any $y \in \mathrm{ObL}(k)$. For a natural system $\mathrm{D}$ on $\int_{\mathbf{K}} \mathrm{L}$ and an object $k$ of $\mathbf{K}$, we have the induced natural system $i_{k}^{*} \mathrm{D}$ on $\tilde{\mathrm{L}}(k)$. Since $\left(l_{k}, r_{k}\right)$ is an adjoint pair from $\tilde{\mathrm{L}}(k)$ to $\mathrm{L}(k)$, we can apply Proposition 2.3 setting $\mathrm{E}_{k}=i_{k}^{*} \mathrm{D}$. One has $\overline{\mathrm{E}}_{k}=\mathrm{D}_{k}$, where $\mathrm{D}_{k}$ is a natural system on $\mathrm{L}(k)$ given by

$$
\mathrm{D}_{k}\left(x_{0} \stackrel{\xi}{\rightarrow} x_{1}\right)=\mathrm{D}\left(\left(k, x_{0}\right) \stackrel{\left(i d_{k}, \xi\right)}{\longrightarrow}\left(k, x_{1}\right)\right) .
$$

On the other hand

$$
\tilde{\mathrm{E}}_{k}(\beta, \psi)=\mathrm{D}\left(\alpha, \mathrm{L}\left(\alpha^{\prime}\right)(\psi)\right)
$$

for any morphism $(\beta, \psi):(\alpha, x) \rightarrow\left(\alpha^{\prime}, x^{\prime}\right)$ in $\tilde{\mathrm{L}}(k)$. Hence Proposition 2.3 implies

$$
\mathrm{H}^{*}\left(\mathrm{~L}(k), \mathrm{D}_{k}\right) \simeq \mathrm{H}^{*}\left(\tilde{\mathrm{L}}(k), \tilde{\mathrm{E}}_{k}\right) .
$$

On the other hand there is a natural transformation $i_{k}^{*} \mathrm{D} \rightarrow \tilde{\mathrm{E}}_{k}$ of natural systems on $\tilde{\mathrm{L}}(k)$. We will say that the natural system $\mathrm{D}$ is $h$-local provided, for any object $k$ in $\mathbf{K}$, the induced homomorphism in cohomology $\mathrm{H}^{*}\left(\tilde{\mathrm{L}}(k), i_{k}^{*} \mathrm{D}\right) \rightarrow \mathrm{H}^{*}\left(\tilde{\mathrm{L}}(k), \tilde{\mathrm{E}}_{k}\right)$ is an isomorphism. If this holds then $\mathrm{H}^{*}\left(\tilde{\mathrm{L}}(k), i_{k}^{*} \mathrm{D}\right) \cong \mathrm{H}^{*}\left(\mathrm{~L}(k), \mathrm{D}_{k}\right)$ thanks to isomorphism (11). Thus in this case the functors $\mathbb{H}^{*}\left(\mathrm{~L}(),. \mathrm{D}_{(.)}\right)$and $\mathbb{H}^{*}\left(\tilde{\mathrm{L}}(),. i_{(.)}^{*} \mathrm{D}\right)$ are isomorphic. By Theorem 4.1 we obtain the following result. 
THEOREM 5.1 Let $\mathbf{K}$ be a small category and let $\mathrm{L}: \mathbf{K} \rightarrow \mathbf{C A T}$ be a functor. Assume $\mathrm{D}$ is a natural system on $\int_{\mathbf{K}} \mathrm{L}$ which is h-local. Then there is a first quadrant spectral sequence

$$
\mathrm{E}_{2}^{p q}=\mathrm{H}^{p}\left(\mathbf{K}, \mathbb{H}^{q}\left(\mathrm{~L}(.), \mathrm{D}_{(.)}\right)\right) \Rightarrow \mathrm{H}^{p+q}\left(\int_{\mathbf{K}} \mathrm{L}, \mathrm{D}\right),
$$

where $\mathbb{H}^{q}\left(\mathrm{~L}(),. \mathrm{D}_{(.)}\right): \mathbf{K}^{\mathrm{op}} \rightarrow \mathbf{A b}$ sends $k \in \mathrm{Ob} \mathbf{K}$ to $\mathrm{H}^{q}\left(\mathrm{~L}(k), \mathrm{D}_{k}\right)$ and $\mathrm{D}_{k}$ is a natural system on $\mathrm{L}(k)$ given by

$$
\mathrm{D}_{k}\left(x_{0} \stackrel{\xi}{\rightarrow} x_{1}\right)=\mathrm{D}\left(\left(k, x_{0}\right) \stackrel{\left(i d_{k}, \xi\right)}{\longrightarrow}\left(k, x_{1}\right)\right) .
$$

REMARK 5.2 Assume D is a natural system such that the morphism $i_{k}^{*} \mathrm{D} \rightarrow \tilde{\mathrm{E}}_{k}$ of natural systems is an isomorphism. Then obviously $\mathrm{D}$ is h-local. In this case we will say that $\mathrm{D}$ is local. Then $\mathrm{D}$ is local if and only if for any morphism $\left(k_{0}, x_{0}\right) \stackrel{(\alpha, \xi)}{\longrightarrow}$ $\left(k_{1}, x_{1}\right)$ in $\int_{\mathbf{K}} \mathrm{L}$ and any arrow $\beta: k_{0} \rightarrow k_{1}$, the induced homomorphism

$$
\left(\beta, i d_{\mathrm{L}(\beta)\left(k_{1}\right)}\right)_{*}: \mathrm{D}(\alpha, \xi) \rightarrow \mathrm{D}\left(\left(\beta, i d_{\mathrm{L}(\beta)\left(k_{1}\right)}\right)_{*}(\alpha, \xi)\right)
$$

is an isomorphism. It is now clear that this condition automatically holds, for instance, when $\mathbf{K}$ is a groupoid, or $\mathbf{K}$ is arbitrary and D is of the form

$$
\mathrm{D}\left(\left(k_{0}, x_{0}\right) \stackrel{(\alpha, \xi)}{\longrightarrow}\left(k_{1}, x_{1}\right)\right)=\mathrm{M}\left(k_{0}, x_{0}\right)
$$

where $\mathrm{M}:\left(\int_{\mathbf{K}} \mathrm{L}\right)^{\mathrm{op}} \rightarrow \mathbf{A b}$ is a functor.

REMARK 5.3 Let $k$ be a field and let $\mathcal{C}$ be a $k$-linear category. If $G$ is a group acting freely on $\mathcal{C}$, one can consider the quotient category $\mathcal{C} / G$. Now, for any locally finite $\mathcal{C} / G$-bimodule $\mathcal{M}$, there is a spectral sequence

$$
\mathrm{E}_{2}^{p, q}=H^{p}\left(G, H^{q}(\mathcal{C}, \mathrm{L} \mathcal{M})\right) \Rightarrow H^{p+q}(\mathcal{C} / G, \mathcal{M}),
$$

see [CR, Theorem 3.11]. Moreover, the skew category $\mathcal{C}[G]$ defined in $[\mathrm{CM}$ is equivalent to the quotient category $\mathcal{C} / G$, see $[\mathrm{CM}$. Theorem 2.8]. Assume now that $\mathbf{K}$ is the one object category associated to the group $G$. Define $\mathrm{L}$ as the functor $\mathbf{K} \rightarrow \mathbf{C A T}$ that sends the unique object to the category $\mathcal{C}$, and morphisms in $\mathbf{K}$ corresponding to the $G$-action. One easily sees that for the corresponding $k$-linear span $k[\mathcal{C}]$ one has an isomorphism of $k$-linear categories

$$
k[\mathcal{C}][G] \simeq k\left[\int_{\mathbf{K}} \mathrm{L}\right] .
$$

Thus the spectral sequence constructed in [CR gives the spectral sequence constructed in 5.1 for such $\mathbf{K}$, when coefficients are bifunctors. 
REMARK 5.4 Let $\mathbf{K}$ be the one object category associated to a group $G$ and let $\mathrm{L}: \mathbf{K} \rightarrow \mathbf{C A T}$ be a functor, that is, a category $\mathbf{C}$ with a $G$-action. For any natural system D on $\int_{\mathbf{K}} \mathrm{L}$, Theorem 5.1 says that we have a spectral sequence

$$
\mathrm{E}_{2}^{p, q}=H^{p}\left(G, H^{q}(\mathbf{C}, \mathrm{D})\right) \Rightarrow H^{p+q}\left(\int_{\mathbf{K}} \mathrm{L}, \mathrm{D}\right) .
$$

In this particular case, an alternative proof can be given using a well-known theorem of Eilenberg-Zilber-Cartier DP that says that the diagonal of a bicosimplicial abelian group is homotopy equivalent to the total complex of the associated bicomplex. Consider the bicomplex $C^{* *}=C^{*}\left(G, F^{*}(\mathbf{C}, \mathrm{D})\right)$, where $C^{*}(G, M)$ denotes the Eilenberg-Mac Lane cochains of the group $G$ with coefficients in a $G$-module $M$, and the $G$-action on the cochain complex $\mathrm{F}^{*}(\mathbf{C}, \mathrm{D})$ is given by

$$
f^{s}\left(\alpha_{1}, \cdots, \alpha_{p}\right)=(s, i d)_{*}\left(s^{-1}, i d\right)^{*} f\left(s^{-1} \alpha_{1}, \cdots, s^{s^{-1}} \alpha_{p}\right)
$$

for any $s \in G$. An explicit isomorphism from the diagonal of $C^{* *}$ to $\mathrm{F}^{*}\left(\int_{\mathbf{K}} \mathrm{L}, \mathrm{D}\right)$ is given as follows. For any $g_{1}, \cdots, g_{p} \in G, \alpha_{1}, \cdots, \alpha_{p} \in \mathbf{C}$, we set

$$
\begin{gathered}
\phi^{p}(f)\left(\left(g_{1}, \alpha_{1}\right) \circ \ldots \circ\left(g_{p}, \alpha_{p}\right)\right)= \\
=\left(g_{1} \cdots g_{p}, i d\right)^{*} f\left(g_{1}, \ldots, g_{p}, \alpha_{1},{ }^{g_{1}} \alpha_{2}, \ldots,{ }^{g_{1} \cdots g_{p-1}} \alpha_{p}\right) .
\end{gathered}
$$

ACKNOWLEDGEMENTS. This paper was written while the authors were visiting the Université de Montpellier II. They want to express their gratitude to Claude Cibils and Daniel Guin for their hospitality.

\section{References}

[BW] H. J. Baues, G. Wirsching. Cohomology of small categories. J. Pure Appl. Algebra 38, no. 2-3, 187-211 (1985).

$[\mathrm{CM}]$ C. Cibils, E. Marcos. Skew categories, Galois coverings and smash product of a $k$-category. To appear in Proc. Amer. Math. Soc.

[CR] C. Cibils, M. J. Redondo. Cartan-Leray spectral sequence for Galois coverings of linear categories. J. of Algebra. 284, 310-325 (2005).

[DP] A. Dold, D. Puppe. Homologie nicht-additiver Funktoren. Anwendungen. Ann. Inst. Fourier Grenoble 11, 201-312 (1961).

[FFPS] V. Franjou, E. M. Friedlander, T. Pirashvili, L. Schwartz. Rational Representations, the Steenrod Algebra and Functor Homology. Panoramas et Synthèses, vol. 16, Soc. Math. France, Paris, xxii+132 pages (2003).

[M] F. Muro. On the functoriality of cohomology of categories. arXiv math.CT/0411478 
[P] T. Pirashvili. Category of Eilenberg-Mac Lane fibrations and cohomology of Grothendieck constructions. Comm. Algebra 21, no. 1, 309-341 (1993).

[Q] D. Quillen. Higher algebraic $K$-theory. I. Algebraic $K$-theory, I: Higher $K$ theories (Proc. Conf., Battelle Memorial Inst., Seattle, Wash., 1972), pp. 85147. Lecture Notes in Math., Vol. 341, Springer, Berlin (1973).

[SGA1] Revêtements étales et groupe fondamental. Séminaire de Géomètrie Algébrique du Bois Marie 1960-1961 (SGA 1). Dirigé par Alexandre Grothendieck. Augmenté de deux exposés de M. Raynaud. Lecture Notes in Mathematics, Vol. 224. Springer-Verlag, Berlin-New York. xxii+447 pp. (1971)

[T] R. W. Thomason. Homotopy colimits in the category of small categories. Math. Proc. Cambridge Philos. Soc. 85, no. 1, 91-109 (1979). 